\title{
A FRACTILE-BASED RELIABILITY STRUCTURAL DESIGN
}

\author{
By Fumio NISHINO*, Akio HASEGAWA**, Chitoshi MIKI*** \\ and Yozo $F U J I N O * *$
}

\section{INTRODUCTION}

Although the probability of failure is theoretically a logical measure of structural safety, it is determined only when statistical information is known for all of the random variables that influence the attainment of limit states of a structure. For the reasons that available statistical information is limited at present and that the failure probability is sensitive to the tail of distributions, it is impractical to measure structural safety by the failure probability. The method of the second moment has been introduced as a practical alternative that makes use of distribution-free statistics of data that are inherently insensitive to distribution shapes. Subsequently it has attracted wide attention of structural engineers and code writers ${ }^{1) \sim 7)}$ and is adopted as a statistical basis for numerous design code systems or has influenced the change of the allowable stress design format to the partial safety factor format ${ }^{8) \sim 12)}$. In the second moment method, the reliability level is measured by the so-called safety indices, that are the functions of the means and the coefficients of variation of random variables influencing the attainment of limit states. Means and coefficients of variation are insensitive to extremely rare events. This is the major advantage of the second moment method under the conditions of limited information but with the sacrifice of accuracy in terms of the failure probability. This is contrary to the concept that structural design is to achieve a specified failure probability. The failure probability may not be consistent but may vary widely even if a design is accomplished under a specified constant index. Within the frame work of the second moment method, a better correlation can

* Member of JSCE, Prof. of Civ. Engrg., Univ. of Tokyo

** Member of JSCE, Assoc. Prof. of Civ. Engrg., Univ. of Tokyo

*** Member of JSCE, Assoc. Prof. of Civ. Engrg., Tokyo Inst. of Tech. be achieved by incorporating information on distribution shapes ${ }^{6)}$. This is possible only when sufficient information is available on distribution shapes and at the sacrifice of the simplicity of the second moment method.

Another drawback of the second moment method is that all of the random variables are treated on equal basis, disregarding the difference of available amount of statistical information among variables. For example, number of experimental data on the structural behavior of real size specimens are very few, while numerous data are available for strength of materials. Further, there exist some variables on which it is possible to certain extent to favor a particular statistical distribution by probabilistic reasoning or by engineering judgement.

In the second moment method, the design values of load and resistance, that is, the load and resistance factors in the terminology of the method, can not be determined independently. Independent determination of the so-called partial safety factors can only be accomplished by a mathematical approximation and by an optimization procedure on various structures and locations of the structures.

A possible and equally simple alternative to the second moment method is a design format in which structures are designed such that forces generated by very high specified loads are compared with the forces required to attain limit states evaluated with specified low values of resistance. In fact, this is similar to most of the conventional design formats. The format proposed by Ang and $\mathrm{Amin}^{4}$ ) is similar to this format in fundamental concept, however, there exists a significant difference in a way that design values are determined. The values on load effect and resistance are not determined separately but interrelated as are the case of the second moment method.

The aim of this paper is to present the statistical basis of this design format that utilizes the low values of resistance population and the high values of load effect and is to make clear that this format is rather flexible than the second 
moment method. Whenever there is a reason to favor a particular distribution, it can be easily incorporated into the determination of design values to attain more consistent failure probability. The differences in available amounts of statistical information can also be reflected in the selection of design values.

\section{FRACTILE DESIGN METHOD}

The condition that a structure reaches a limit state can be symbolically expressed as

$$
Z^{(i)}=Z^{(i)}\left(r_{1}, r_{2}, \cdots, r_{p}, s_{1}, \cdots, s_{q}, t\right)=1
$$

where the parameters $\left(v_{1}, v_{2}, \ldots, v_{p}\right)$ are random variables that influence strength and other limit states of the structure such as material properties, values of dimensions, and tolerances. The parameters $\left(s_{1}, s_{2}, \ldots, s_{q}\right)$ are random variables that influence load effect such as dead loads and live loads. The variable $t$ represents all other variables that are not included in $r$ and $s$ by the reasons that their treatment in structural analysis is difficult and/or their properties are uncertain. The superscript $i$ denotes the type of limit states that have to be considered in design such as static ultimate state, excessive deflection, unfavorable vibration, fatigue failure, long term variation due to creep and so on. For each of these limit states, there exists a single function $Z$. In the following development, the superscript $i$ is omitted for simplicity with the understanding that all of the following discussions are applicable to each of the limit states.

The parameter $t$ is disregarded at this stage and only the parameters $r$ and $s$ are considered. There may be a design method in which Eq. (1) is utilized as it is. An earthquake resistant design which uses a simulation of a whole structural model during earthquake motion is one of the example. In general, however, structural design does not make use of Eq. (1) in its general form. It is common practice that forces (or stresses) generated by estimated loads at a member or a cross section in a structure are compared with forces (or stresses) required for attainment of a limiting state. This is equivalent to the assumption that $Z$ is a functional of two functions, one is a function of only $r$ 's and the other is a function of $s$ 's. The former is called resistance and the latter load effect, designated by $R$ and $S$, respectively. Then Eq. (1) can be written for a member or a cross section as

$$
Z=Z\left\{R\left(v_{1}, v_{2}, \cdots, v_{p}\right), S\left(s_{1}, \cdots, s_{q}\right)\right\}=1 \cdots \cdots(2)
$$

Some of the random parameters (e.g. dimensions) are common to both $R$ and $S$ and thus $R$ and $S$ may not be independent. Some of the parameters are time-dependent.
There are many variations of the expression of the functional $Z$ that appear in structural design. One of the examples is the so-called interaction formula expressed as

$$
Z=\sum_{j}\left[\left\{\frac{S\left(s_{1}, s_{2}, \cdots, s_{q}\right)}{R\left(v_{1}, v_{2}, \cdots, r_{p}\right)}\right\}^{m}\right]_{j}
$$

where $m$ 's are usually constants. The simplest and the most popular formula of Eq. (3.a) is the expression with $m=j=1$

$$
Z=\frac{S\left(s_{1}, \cdots, s_{q}\right)}{R\left(r_{1}, \cdots, v_{p}\right)}
$$

For the purpose of illustration, only Eq. $(3 \cdot \mathrm{b})$ is considered in the following development.

The evaluation of the function $R$ in Eq. (3) in terms of its variables may be called strength analysis and that of $S$ stress analysis. One of the random variables $r$ and that of $s$, say $v_{n}$ and $s_{n}$, may represent random model uncertainties in $R$ and $S$, when they need to be considered, defined by

$$
\left.\begin{array}{l}
r_{n}=\frac{\text { actual strength }}{\text { predicted strength }} \\
s_{n}=\frac{\text { actual load effect }}{\text { predicted load effect }}
\end{array}\right\} \cdots \cdots \cdot(4 \cdot \mathrm{a}, \mathrm{b})
$$

A limit state considered in a design is exceeded when $Z>1$, provided that the disregarded parameter $t$ plays no important role. The probability of failure $P_{f}$ is then

$$
\begin{aligned}
P_{f} & =\operatorname{Pr}(Z>1)=\operatorname{Pr}(R-S<0) \\
& =\int_{-\infty}^{\infty}\left\{\int_{-\infty}^{S} f_{R}(R / S) d R\right\} f_{S}(S) d S
\end{aligned}
$$

where $f_{R}(R / S)$ is the conditional probability distribution function of $R$ and $f_{S}(S)$ is the probability density function of $S$. The reliability is defined by $1-P_{f}$. In most cases, $P_{f}$ is a function of the reference period being considered.

In order to utilize the reliability based structural design with statistically defined design values of random variables $R$ and $S$, the deterministic parameters $R^{*}$ and $S^{*}$ are defined by fractiles of distributions as

$$
\operatorname{Pr}\left(R<P^{*}\right)=e_{R}, \quad \operatorname{Pr}\left(S>S^{*}\right)=e_{S} \cdots \cdots(6 \cdot \mathrm{a}, \mathrm{b})
$$

where $e_{R}$ and $e_{S}$ are constants of small values (e.g. $10^{-2}$ through $10^{-6}$ ) and the superscript asterisk indicates design values. It is proposed to design a structure utilizing only the statistically defined parameters $R^{*}$ and $S^{*}$ to satisfy the following equations when the functional $Z$ is expressed by Eq. $(3 \cdot \mathrm{b})$

$$
S^{*} / R^{*} \leqq 1 \cdots
$$

The probability of failure of a structure dissatisfying Eq. (7) is clearly a function of specified fractiles $e_{R}$ and $e_{S}$ and shapes of $R$ and $S$ distributions. The fact that the probability of failure is 
a function of the fractiles for $R$ and $S\left(e_{R}\right.$ and $\left.e_{S}\right)$ suggests that a combination of fractiles $e_{R}$ and $e_{S}$ can be a measure of the degree of reliability of a structure designed by Eq. (7). The fractiles have to be determined in such a way that the failure probability of a structure designed by Eq. (7) is less than a specified target value.

In any practical structural design, the resistance $R$ and the load effect $S$ are not functions of single variable. The load effect at a structural element is a function of loading acting at various locations of the structure. The loading at any single location is composed of various loadings such as dead load, live loads, temperature effects and so on. Hence, the load effect $S$ for a structural element may be expressed as

$$
S=S\left(\Sigma s^{1}, \Sigma s^{2}, \cdots, \Sigma s^{j}, \cdots\right)
$$

where $\Sigma_{s}^{j}$ indicates all of the loadings working at location $j$ of the structure. For a structure that responds linearly to loadings, Eq. (8) takes a simple form and can be written as

$$
S=\sum_{j}\left(c_{1}{ }^{j}{ }^{j}{ }^{j}+c_{2}{ }^{j} S_{2}^{j}+\cdots+c_{q}^{j} S_{q}^{j}\right)
$$

where $c_{i}{ }^{j}$ is deterministic influence coefficient for loading $s i$ at location $j$. For a structure for which model uncertainty can not be disregarded, the influence coefficients may be treated as random variables to include this effect, or additional random variables such as defined in Eq. (4.b) may be introduced to each influence coefficient.

The resistance $R$ of a member is often expressed by a product of functions

$$
R=R_{1} R_{2} \cdots R_{m}
$$

where $R_{i}$ are functions of $r$.

\section{FRACTILE AND FAILURE PROBABILITY}

In the proposed statistical design method of Eq. (7), the structural safety is not directly evaluated by the failure probability but the reliability level is defined by the fractiles. In this regard, the probabilistic basis is similar to the second moment design method in which reliability level is defined by the so-called safety indices. Since the failure probability is not the direct target in these semi-probabilistic design methods, correlation between failure probability and reliability indices such as the safety indices or the fractiles is one of the most important factors in the evaluation of superiority as a design method. Semi-probabilistic design methods can be recognized as rational and practical substitutes to the method based on exact probabilistic failure analysis, only when good correlation exists between the measure of reliability levels and the failure probability.
For the ease of discussion, the fractile of resistance $e_{R}$ is selected to be equal to that of load effect $e_{S}$, that is $e_{R}=e_{S}=e$. When $R$ and $S$ are statistically independent, the failure probability is bounded by the fractile ${ }^{4}$ as

$$
e^{2}<P_{f}<2 e-e^{2}
$$

Equation (11) does not guarantee a good correlation. The variation of $P_{f}$ would be large for a fixed target of fractile. In order to examine the correlation, numerical calculations are performed. Noting the functional relation of Eq. (9) for load effect and that of Eq. (10) for resistance, the central limit theorem suggests that the distributions of load effect and resistance may be closer to normal and logarithmic normal distribution, respectively. Nevertheless, the types of distribution of the random variables $R$ and $S$ that are functions of a number of basic random variables can not generally be identified. Because of this inherent variability of distribution functions, numerical calculations are performed selecting a variety of mathematically defined distribution functions for $R$ and $S$, namely normal distributions for both $R$ and $S$ (designated by $N / N)$, logarithmic normal distributions for both $R$ and $S(\mathrm{LN} / \mathrm{LN})$, types 3 and 1 extreme distributions for $R$ and $S$, respectively, (EX3/ EX1), and types 3 and 2 for $R$ and $S$ (EX3/EX2). Two combinations of coefficients of variation are selected for $R$ and $S$, one being 0.05 and 0.10 , respectively, and the other being 0.10 and 0.20 . Specifying the value of fractile and making use of Eq. (7), the failure probability can be evaluated by Eq. (5). Numerical results are presented in Fig. 1. The lines $e^{2}$ and $2 e-e^{2}$ are the boundaries of the failure probability due to Eq. (11).

Similar numerical calculations are performed for the second moment design method. The safety index that is invariant to mechanical formulations is

$$
\beta=\left(\frac{\bar{R}}{\bar{S}}-1\right) / \sqrt{\left(\frac{\bar{R}}{\bar{S}}\right)^{2} V_{R}{ }^{2}+V_{S^{2}}}
$$

where the bar on top of $R$ and $S$ indicates mean values of $R$ and $S$ distributions and $V_{R}$ and $V_{S}$ are the corresponding coefficients of variation. Specifying coefficients of variation and the safety index, Eq. (12) gives $\bar{R} / \bar{S}$, with which the failure probability can be evaluated by Eq. (5). Failure probability is numerically evaluated and the results are presented in Fig. 2 .

Figure 1 shows that, within the trial combinations of distribution functions for $R$ and $S$, the fractile necessary to attain failure probability of order of $10^{-6}$ is $10^{-4}$, and the variation of failure probability for this fractile is of the order of 10 , that is, when the fractile is $10^{-4}, P_{f}$ is 


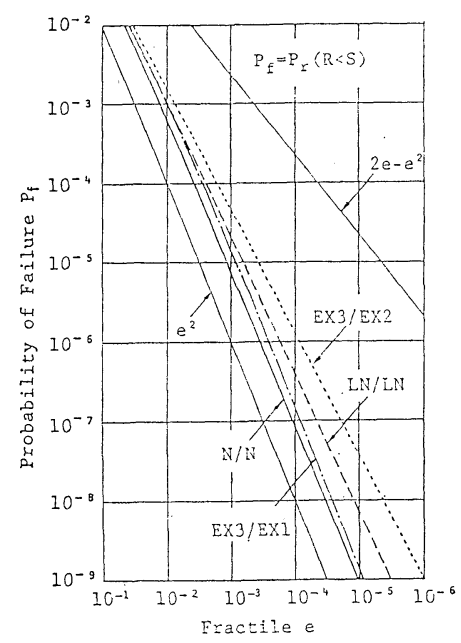

(a) $V_{R}=0.05$ and $V_{S}=0.10$

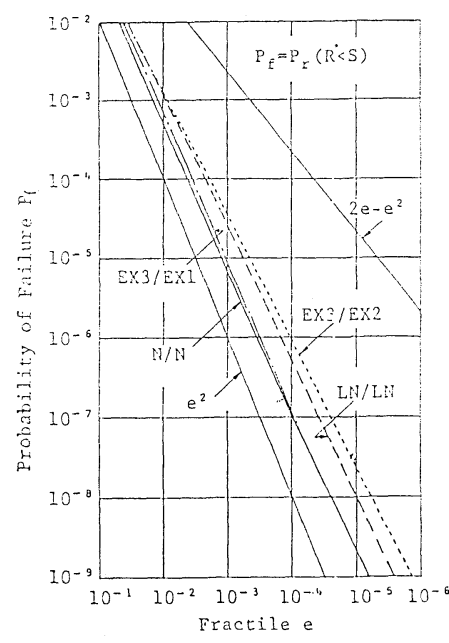

(b) $V_{R}=0.10$ and $V_{S}=0.20$

Fig. 1 Correlation between Fractile and Failure Probability

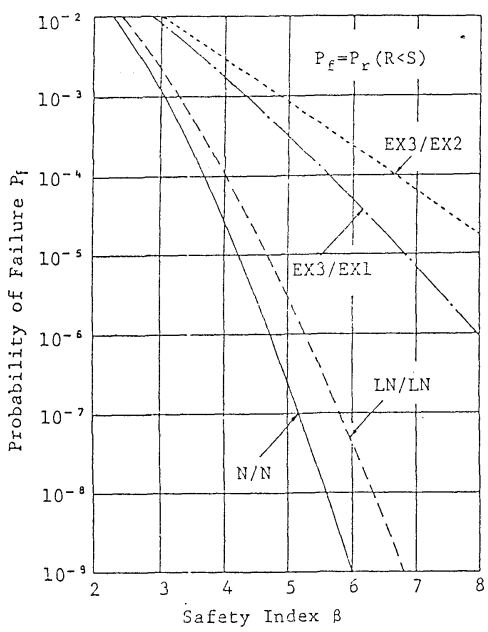

(a) $V_{R}=0.05$ and $V_{S}=0.10$

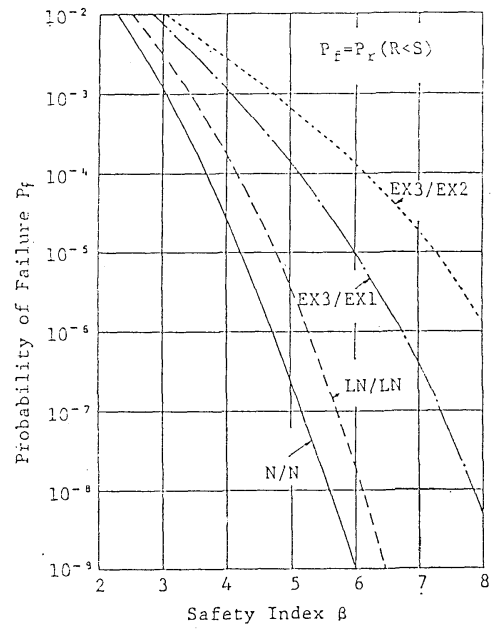

(b) $V_{R}=0.10$ and $V_{S}=0.20$

Fig. 2 Correlation between Safety Index and Failure Probability

within $10^{-6}$ to $10^{-7}$. On the other hand, in the design by the second moment method, the variation of failure probability is much larger for a specified safety index level. For example, for a specified safety index of 6.5 that is necessary to attain $P_{f}$ of order of $10^{-4}$ as can be seen in Fig. 2, the variation of $P_{f}$ is of the order of $10^{-5}$ from $10^{-4}$ to $10^{-9}$. For a smaller target failure probability, it is necessary to specify a larger safety index, for which variation of $P_{f}$ for various combinations of distribution shapes is even larger. Comparison of the numerical results presented in Figs. 1 and 2 asserts that the fractile is far more closely related to the failure probability than the safety index of Eq. (12) and hence from a statistical point of view, the proposed design method of Eq. (7) is much superior to the second moment design method, provided that the deterministic parameter $R^{*}$ and $S^{*}$ can be determined accurately to satisfy their definition of Eq. (6).

\section{LOAD EFFECTS}

Design values $s^{*}$ of loading $s$ are deterministic parameters representing the random variables $s$ in a design process that satisfy

$$
\operatorname{Pr}\left\{S\left(\Sigma_{S^{1}}, \Sigma_{S^{2}}, \cdots\right)>S\left(\Sigma_{S^{* 1}}, \Sigma_{s}{ }^{* 2}, \cdots\right)\right\} \leqq e_{S}
$$


where $\Sigma_{s}^{* j}$ indicates all of the deterministic loadings working at location $j$ of a structure. Assume that model uncertainty need not be considered and that load effect at one location is expressed by Eq. (9). Two simple examples, dead load and dead and live load combination, are discussed for the expression of the design values of load effect.

\section{(1) Dead Load}

Since dead load $S_{D}$ is the only one variable, Eq. (9) becomes

$$
S=\sum_{j} c^{j} S D^{j}
$$

The main factor contributing to the variation of dead load $S_{D}$ is the dimension of structural elements, especially the thickness of component elements. For a very short beam made of a single rolled shape, there may be six dimensions that are regarded as independent random variables. They are three thicknesses for three component plates and, though not important, widths of two flanges and web. For welded built-up shapes, widths may be controlled independently at least at two sections, thus the number of independent dimensions may increase to nine. Steel bridges of reasonable span length may be fabricated with elements cut from numerous rolled shapes and plates. The thickness of each element or of elements from each role is a random variable contributing to dead load. Similarly, innumerable measurements are necessary in formworks for casting reinforced concrete structures. Even for casting $\mathrm{T}$-shaped beams, at least six measurements of thickness may be necessary for a cross section, two for web and two for each side of flange and hence, for one length of form, there exist at least twelve thickness measurements that are the basic independent variables to dead load effect.

From this consideration, it can be understood that, for a practical structure of reasonable scale and importance, the range $j$ of the summation in Eq. (14) for the expression of dead load effect is very large. It is known that, with increasing number of $j$, the distribution of $S$ approaches normal distribution, regardless of the distributions of each random variable $S_{D}$, with the mean $\bar{S}$ expressed by the mean of each independent variable $\bar{s}_{D}$ as

$$
\bar{S}=\sum_{j} c^{j} \bar{S}_{D^{j}}
$$

and the coefficient of variation of $S$ decreasing roughly proportional to $1 / \sqrt{ } \bar{j}$.

For dead load effect, it is reasonable to assume that the distribution of $S$ is normal for a design of practical structures and hence the deterministic parameter $S^{*}$ defined by Eq. $(6 \cdot \mathrm{b})$ is given as

$$
S^{*}=\bar{S}\left\{1 \pm k\left(e_{S}\right) V_{S}\right\}
$$

where $k\left(e_{S}\right)$ is a function of fractile $e_{s}$ that can be found in the table of standard normal distribution. When $e_{S}$ is equal to $10^{-3}, 10^{-4}$, and $10^{-5}$, $k\left(e_{S}\right)$ is equal to $3.10,3.72$, and 4.27 , respectively. It has been reported ${ }^{13), 14)}$ that the coefficients of variation of thickness of concrete slab is, at most, of the order of 0.06 and that of thickness of steel plates is of the order of 0.02 . The coefficients of variation of width would be much smaller.

For the number of independent measurements exceeding 100 or more, which is not unrealistic in practical construction work, the coefficient of variation of dead load effect would be less than one tenth of the coefficients of variation of measurements. Hence even for a fractile of $10^{-3}$ or $10^{-4}$, the design parameter $S^{*}$ is different from mean value $\bar{S}$ only by a few percents at most as shown in Table 1. This difference is within the tolerable range of error in the structural design process, at least at present state of design, and hence may be neglected. If this is so, the mean value $\bar{S}$ can serve the role of the design parameter $S^{*}$ and hence

$$
S^{*} \approx \bar{S}
$$

The design values of the basic random variables which satisfy the requirement of Eq. (13) are generally very high loads when their influence coefficient is positive and very low loads when it is negative. Obviously from the linear property of Eq. (14), the use of the mean value of dead load $\bar{s}_{D}$ leads to the mean value of the load effect $\bar{S}$ that is equal to $S^{*}$ within the tolerable error. Therefore, the design value of dead load $S D^{*}$ can be defined by the mean value. Noting in Eq. (17) that $\bar{S}$ represents the load effect of both high and low sides, it is obvious that this mean value can be used as a design value at any location regardless of the sign of influence coefficients. It has to be noted that some specifications allow a tolerance of dimensions only in positive side. If this is the case, the mean values of dead load may not be equal to the values evaluated based on nominal dimensions specified in design, but they may be biased. The design

Table 1 Deviations of Design Values from Mean

\begin{tabular}{c|c|c|c|c}
\hline $\begin{array}{c}\text { Target } \\
\begin{array}{c}\text { Fractile } \\
e_{R}\end{array}\end{array}$ & $k\left(e_{R}\right)$ & $\begin{array}{c}\text { C.O.V. of } \\
\text { Dead } \\
\text { Load }\end{array}$ & $\begin{array}{c}\text { C.O.V. of } \\
\text { Load Effect } \\
\text { for } j=100\end{array}$ & $\begin{array}{c}k\left(e_{R}\right) V_{S} \\
\text { for } j=100\end{array}$ \\
\hline $10^{-3}$ & 3.10 & & & $0.019 \sim 0.006$ \\
$10^{-4}$ & 3.72 & $0.06 \sim 0.02$ & $0.006 \sim 0.002$ & $\begin{array}{l}0.022 \sim 0.007 \\
10^{-5}\end{array}$ \\
\hline
\end{tabular}


values are, then, to be evaluated based on target dimensions.

\section{(2) Dead and Live Load Combination}

The load effect $S$ for combined dead and live loads can be written as

$$
S=\sum_{j} c^{j}\left(s D^{j}+s L^{j}\right)
$$

where $s L^{j}$ indicates the distribution of the maximum or the minimum live load during a fixed length of period that acts at location $j$. Substitution of Eq. (17) for dead load into Eq. (18) results in

$$
S=S_{D} *+\sum_{j} c^{j} S_{L}^{j}
$$

where $S_{D} *$ is the design value of dead load effect.

Consider the simplest case that the magnitude of $s_{L}$ at an instant can be assumed constant over the domain where it acts. Live load over a short span bridge is an example. For this case, the summation over $j$ may be changed into integration over the domain (indicated by $L$ ) where live load acts, with which Eq. (19) can be written as

$$
S=S_{D} *+C_{L}
$$

where

$$
C=\int_{L} c(x) d x
$$

Substituting Eq. (20) into Eq. (6.b), the deterministic design value of the live load $S L^{*}$ is defined by

$$
\operatorname{Pr}\left\{S>\left(S_{D} *+C S_{L}^{*}\right)\right\}=e_{S}
$$

Since $S_{D} *$ is a deterministic value, Eq. (20) is regarded as a linear transformation between $S L$ and $S$. From the property of linear transformation, Eq. (22) is expressed as follows from which the design value of the live load is determined for a given fractile for load effect $S$.

$$
\begin{aligned}
\operatorname{Pr} & \left\{S>\left(S_{D}^{*}+C s_{L}^{*}\right)\right\} \\
& = \begin{cases}\operatorname{Pr}\left(s_{L}>s_{L} *\right) & \text { when } C>0 \\
\operatorname{Pr}\left(s_{L}<s_{L}^{*}\right) & \text { when } C<0\end{cases}
\end{aligned}
$$

It is noted that, for a gravity live load, the minimum live load distribution is identically equal to zero and hence Eq. $(23 \cdot \mathrm{b})$ results in

$$
S_{L}^{*}=0
$$

With increasing width and span length, live load intensity at an instant can no longer be uniform. Rather, live loads at different locations are independent variables of identical distributions. Taking a reasonably small area over the surface where live loads act as one unit, the load effect can be evaluated by Eq. (19) as the sum of the live loads acting over these unit areas. The same discussion follows for the mean and the coefficient of variation of load effect as for dead load. As a result, when live load is modeled into an uniformly distributed loading, the magnitude of design value $s L^{*}$ decreases with increasing span length and width and, at their limits, approaches to the mean value of the maximum live load distribution.

\section{RESISTANCE}

Consider the case when resistance is expressed by Eq. (10). In view of this expression and Eq. $(6 \cdot a)$, the deterministic parameters $R_{i} *$ of random variables that constitute components of resistance are defined by

$$
\operatorname{Pr}_{r}\left(R_{1} R_{2} \cdots R_{m}<R_{1} * R_{2} * \cdots R_{m} *\right)=e_{R}
$$

The probabilistic definition of each deterministic parameter is arbitrary as long as the other parameters are selected such that Eq. (25) be satisfied. One of the simplest is to define all of $R_{i} *$ by the same fractile $e c$ as

$$
\operatorname{Pr}\left(R_{1}<R_{1}^{*}\right)=\cdots=P_{r}\left(R_{m}^{*}<R_{m} *\right)=e_{C} \cdots \cdots
$$

The other possibility is to select different fractiles for different variables and define the deterministic parameters by

$$
\operatorname{Pr}_{r}\left(R_{1}<R_{1} *\right)=e_{1}, \cdots, \operatorname{Pr}\left(R_{m}<R_{m} *\right)=e_{m} \cdots
$$

The fractiles have to be determined by the condition that Eq. (25) is satisfied with the deterministic parameters defined by Eqs. (26) or (27).

As an example of the expression of resistance, the ultimate static strength $P_{u}$ of a pinned end metal column may be expressed as

$$
\begin{aligned}
P_{u} & =P_{u}\left(A, \sigma_{y}, x_{1}, \cdots, x_{n}\right) \approx A \sigma_{y} g\left(x_{1}{ }^{\prime}, \cdots, x_{n}{ }^{\prime}\right) \\
& \equiv A \cdot \sigma_{y} \cdot\left(\sigma_{u_{b}} / \sigma_{y}\right) \quad \cdots \cdots \cdots \cdots \cdots \cdots \cdots \cdots \cdots \cdots(28) \cdots \cdots \cdots \cdots \cdots \cdots \cdots \cdots
\end{aligned}
$$

where $A$ is cross sectional area, $\sigma_{y}$ is an appropriate measure of strength of material such as static yield stress or yield stress defined by offset strain, and $g(\cdot)$ is a complex and, in general, nonlinear function of slenderness ratio, residual stress, material nonlinear function of slenderness ratio, residual stress, material nonlinearity, initial imperfections and, perhaps, of some other factors, as denoted by $\left(x_{1}, \ldots, x_{n}\right)$ or $\left(x_{1}{ }^{\prime}, \ldots, x_{n}{ }^{\prime}\right)$. The function of $g(\cdot)$ is often referred to as $\left(\sigma_{u} / \sigma_{y}\right)$, which is the ultimate stress of a column nondimentionalized by $\sigma_{y}$ of the same column and it is treated as one variable. The term $\left(\sigma_{u} / \sigma_{y}\right)$ may be divided into as many components as the number of factors that influence $\sigma_{u}$, however, this effort may not be worthwhile and at present Eq. (28) is one of the most popular expressions for columns with slenderness ratios of practical importance.

For the purpose of illustrating the evaluation of the deterministic parameters, a simple example is presented with resistance expressed by a product of two components as

$$
R=R_{1} R_{2}
$$


Considering an idealized case of a column with well controlled dimensions, cross sectional area may be treated as a deterministic value with sacrifice of little statistical error in the evaluation of ultimate strength. If this is the case, Eq. (28) is an example of Eq. (29), otherwise it can be treated as a case with three components. Because of inherent variability of random variables $R_{i}$, the fractiles to be used in Eqs. (26) or (27) are best determined by numerical experiments. The type of distribution for this resistance with two components depends on the statistical property of each component. Whenever there is an evidence to favor a particular statistical distribution for some of the components of resistance either by a qualitative study of the variable or by statistical data, these distributions can be utilized in the numerical experiments to yield more accurate results. For the components for which distribution is not known or data are too few to determine the shape of distribution, a variety of distributions needs to be assumed.

It has to be noticed that, generally, available statistical information for various components is significantly different, and that the expense or the effort needed to collect statistical information that is not available at present but necessary for the future development varies depending on the components. For example, the statistical data available on $\sigma_{y}$ of Eq. (28) far exceed those on $\left(\sigma_{u} / \sigma_{y}\right)$, and the expense or the effort to collect information is far less for $\sigma_{y}$ than that for $\left(\sigma_{u} / \sigma_{y}\right)$. In view of this difference of available statistical information, it may be natural to select different values of fractile for different components and the deterministic parameters are determined utilizing Eq. (27). A simple numerical example is performed to illustrate this procedure.

For a resistance expressed by Eq. (29), one of the two fractiles, $e_{1}$ and $e_{2}$, can be selected arbitrarily, and then the other is determined so as to satisfy Eq. (25) for a given target fractile of the resistance. As an example, the fractile $e_{1}$ for $R_{1}$ is selected to be equal to the target fractile $e_{R}$ as

$$
e_{1}=e_{R}
$$

Then, the required fractile for $R_{2}$ to satisfy Eq. (25) for various values of $e_{R}$ is numerically evaluated employing logarithmic normal distributions for both $R_{1}$ and $R_{2}$. The results are presented in Fig. 3. Two curves are the results for two different ratios of the coefficients of variation, $V_{2} / V_{1}=0.5$ and 1 .

It can be seen that the required fractile with which the deterministic parameter $R_{2} *$ is deter-

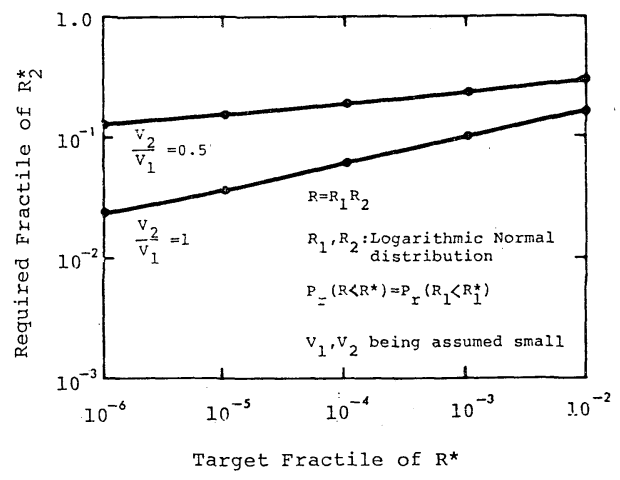

Fig. 3 Required Fractile of $R_{2}{ }^{*}$

mined by Eq. (27) is much larger than a given target fractile. For example, for a given target fractile of $e_{R}=10^{-4}, e_{2}$ is roughly 0.06 for $V_{2} / V_{1}=$ 1 and 0.2 for $V_{2} / V_{1}=0.5$, and for $e_{R}=10^{-3}, e_{2} \approx 0.1$ and 0.3 , respectively. It has been reported ${ }^{14), 15}$ ) that the coefficient of variation of yield stress is of the order of 0.1 and that of column strength $\left(\sigma_{u} / \sigma_{y}\right)$ is of the order of 0.02 for normalized slenderness ratio $\lambda \approx 0.6$, increases to the order of 0.1 for $\lambda \approx 1.0 \sim 1.2$ and decreases with further increase of $\lambda$. Since the coefficient of variation of $\left(\sigma_{u} / \sigma_{y}\right)$ varies with the value of slenderness ratio, the fractile is better determined as a function of slenderness ratio. With the fractile $e_{2}$ so determined, the design value $\left(\sigma_{u} / \sigma_{y}\right)^{*}$ can be determined by Eq. (27).

For this illustration, both $R_{1}$ and $R_{2}$ have been assumed logarithmic normal. In practical application such as specification writing, numerical experiments must be carried out for combinations of distribution types that may represent the distribution of each component. If no evidence is available to favor any particular distribution for a component, as is likely the case for most of the components, then various types of distributions have to be examined for the component. If the distribution shapes are known even only vaguely, the determination of design values $R_{i} *$ by Eq. (27) can be done by taking this information into consideration.

The determination of design values are easier with smaller statistical error, if the fractile to be used in Eq. (27) is larger. This is particularly so when available statistical information is limited. It may be seen from Fig. 3 that this can be accomplished by selecting a smaller fractile for a component with larger coefficient of variation among the components with sufficient statistical data. In many resistance expressions of practical importance, a measure of material strength appears as one of the com- 
ponents. Since the available statistical information is the largest and the coefficient of variation is reasonably large, material strength would be the natural selection for this purpose. By this selection, the design values of other components are to be specified at a relatively low value but not at an extremely low value of the population.

\section{COMPARISON WITH CURRENT DESIGN RULES}

The proposed design method is briefly compared with the existing design rules and specifications. Since design values of the proposed design method resembles to those of the allowable stress design format, design values in the allowable stress design are mainly discussed. Most of the existing allowable stress design rules are the products of historical developments and hence specified design values may not be statistically consistent. Nevertheless, examination of these values may reveal a common ground on which they are based.

Among many loads, there are loads for which the maximum magnitudes are physically limited or can be estimated with great accuracy. The design values specified for this type of load are usually the maximum load with possible safeside approximation or the load close to the maximum within errors of a few percents. Static liquid pressure for the design of water and oil containers, dams, and penstocks is one of the examples. For water, specific gravity of 1.0 is specified. For crude oil and its products, specific gravities vary roughly between 0.85 to 0.89 and 0.65 to 0.97 , respectively, depending on a sort of products. It is specified to design by the specific gravity of a particular product when use of the container is limited to that oil, otherwise to use 1.0 as a safeside approximation. Static train load for the design of railway structures is another example. Weight of passengers is evaluated with the number of seats plus number of standing passengers, nine people per one square meters for all of the remaining area, multiplied by $60 \mathrm{kgf}(588 \mathrm{~N})$. Weight of baggages is also evaluated in a similar way. The weight of cars themselves is evaluated with great accuracy with slight safer bias. Hence design loads for locomotives and passenger cars can be regarded as the physically maximum loads. The weight of a freight car may not be the physical maximum, but strict control is enforced for overloading and hence overloading scarcely occurs unless some sorts of human errors are involved or unless special permission is granted. These design values of live loads seem very similar to the design values considered in this proposed design method.
Design values of dead loads in the allowable stress design are specified by the nominal values with specific gravity of each material if it is well defined, otherwise with slightly conservative values when it is subject to variability such as weight of reinforced concrete slabs and timber ${ }^{16)}$. It has already been discussed that the dead load effect evaluated based on target values of dimensions is very close to the maximum and also to the minimum values. If there exist some differences between the target values of dimensions and those in drawings, slight adjustment may be necessary to consider this bias of dead loads. It has been reported, however, that this difference is generally very small and it can be neglected with a few exceptions ${ }^{14)}$. The adoption of a single nominal value to be used at both locations with positive and negative influence coefficients agrees with the design values of the proposed design method.

There are some exceptions of loads that do not correspond to high values of distributions. One of the typical examples is vehicle loading for the design of Japanese highway bridges ${ }^{16)}$. A weight of 20 tonf $(196 \mathrm{kN})$ is specified as the design value of a single truck, while field measurements indicate that this does not correspond to a high value of the distribution of the largest truck weight. This inconsistency compared with others is due to matters of law, law enforcement, policy and social circumstances. Nevertheless, the design value seems much smaller than the optimum and needs to be corrected. Another example could be wind load. Wind load specified in the Japanese specifications for steel highway bridges $^{16)}$ and steel railway bridges ${ }^{17)}$ corresponds to instantaneous wind velocity of 55 meters per second $(200 \mathrm{~km} / \mathrm{h})$. The probability that the maximum wind velocity will not exceed this velocity during 50 -year period is 0.6 . There may be difference in opinions whether this probability is small enough or a still higher velocity should be considered to ensure a smaller probability.

It has to be noticed that design values in existing codes are not necessarily specified by constant values, but variable values are specified. The vehicle loading for highway bridges is given as a function of span length and width. Two different values are specified for pedestrian loading, one for footpaths and the other for roadways ${ }^{16)}$. Similarly different values are specified for train load for static failure, for fatigue and for combination with wind ${ }^{17)}$. This fact has to be noted by two reasons. One is the presence of probabilistic consideration in the selection of design values and the other is the capability of specifying different design values for different 
limit states and load combinations.

Design values of dead loads in the partial safety factor design format are given by the nominal value times the dead load factors. Table 2 shows the dead load factors given in some design codes for static ultimate limit states ${ }^{9) \sim 12)}$. It is noted that two different factors, one for unfavorable effect and the other for favorable effect, are specified. This differs from the design values in the allowable stress design rules, in which the same design values are specified regardless of the effects. The ratio of the two factors being 1.33 to 1.56 (except BS 5400) is very large and is contrary to the aforementioned discussion. With the variability reported in literature ${ }^{13,14)}$, the ratio should be close to 1.0 for structures of reasonable size and importance and hence the dead load factors specified in these partial safety factor design formats are subject to question from a statistical point of view. The same load factor is specified in the CEB/FIP model code and ACI $318 \operatorname{code}^{8)}$ both for dead load and water pressure. The design value of 1.35 or 1.4 times dead load and/or water pressure is unrealistic. It is certain that this factor is not for variability of loading but for something else. If the factor is for something else, it should be multiplied to the load effect computed for more realistic loading, not to the loading itself. The reason will be discussed later in connection with Eq. (35). The same question arises for the design values of live loads. If the specified loading multiplied by a load factor includes variability other than loading, which is likely the case, this factor should be taken out from the design load and should be multiplied later to the load effect.

Resistance in the allowable stress design is specified in the form of resistance divided by a

Table 2 Load Factors for Nominal Dead Load

\begin{tabular}{|c|c|c|c|}
\hline \multirow[b]{2}{*}{ Code } & \multicolumn{2}{|c|}{ Dead Load Factors } & \multirow{2}{*}{$\begin{array}{c}\text { Unfavorable } \\
\text { Favorable }\end{array}$} \\
\hline & $\begin{array}{c}\text { Unfavorable } \\
\text { Effect }\end{array}$ & $\begin{array}{c}\text { Favorable } \\
\text { Effect }\end{array}$ & \\
\hline $\left.\mathrm{CEB} / \mathrm{FIP}^{1}\right)$ & 1.35 & 1.0 & 1.35 \\
\hline $\mathrm{AASHTO}^{2}$ & 1.3 & $1.3 \times 0.75$ & 1.33 \\
\hline CP 110 & 1.4 & 0.9 & 1.56 \\
\hline BS $5400($ Steel) & $\begin{array}{c}1.05\left(1.10^{3)}\right) \\
\text { and } 1.0\end{array}$ & $\begin{array}{c}\left.1.05\left(1.10^{3}\right)\right) \\
\text { and } 1.0\end{array}$ & 1 \\
\hline BS $\begin{array}{l}5400 \\
\text { (Concrete) }\end{array}$ & $\begin{array}{l}\left.1.15\left(1.20^{3}\right)\right) \\
\text { and } 1.0\end{array}$ & $\begin{array}{l}\left.1.15\left(1.20^{3}\right)\right) \\
\text { and } 1.0\end{array}$ & 1 \\
\hline $\begin{array}{l}\text { Allowable } \\
\text { Stress Design }\end{array}$ & 1 & 1 & 1 \\
\hline $\begin{array}{l}\text { Proposed } \\
\text { Format }{ }^{4)}\end{array}$ & $\gamma\left(1+k V_{S}\right) \approx \gamma$ & $\gamma\left(1-k V_{S}\right) \approx \gamma$ & $\frac{1+k V_{S}}{1-k V_{S}} \approx 1$ \\
\hline
\end{tabular}

1) Applicable also for liquid pressure.

2) Resistance factors being included.

3) Applicable when dead load being not accurately assessed.

4) $\gamma$ being a factor for target over nominal dead load ratio. safety factor of which the value is usually obvious in each code for each limit state and load combination. For example, Japanese specifications for steel railway bridges ${ }^{17)}$ employs, as the values of the safety factor, 1.7 for static failure under major loading and 1.0 for fatigue failure and failure due to earthquake. The specified resistance multiplied by the safety factor is appropriate to compare with.

The design values of resistances against static failure in bending $M$, tension $N_{t}$, and compression $N_{c}$, of a member are given in the specifications for steel structures as

$$
\begin{aligned}
& M^{*}=\sigma_{y} * W^{*}, \quad N_{t}^{*}=\sigma_{y} * A^{*}, \\
& N_{c} *=\sigma_{y} * A^{*}\left(\sigma_{u} / \sigma_{y}\right)^{*}
\end{aligned}
$$

where $\sigma_{y} *$ is the minimum value of yield stress guaranteed in specifications for steel, $A^{*}$ and $W^{*}$ are the nominal values of cross section and section modulus. The expression of Eq. (31.a) is applicable for beams free from lateral instability. The guaranteed minimum value of yield stress corresponds to a very low value of its population. The nominal values of $A$ and $W$ are also located. nearly on, or slightly on a lower side of the mean of the population. Noting that the coefficients of variation of $A$ and $W$ would be of the order of one half or less of that of $\sigma_{y}$ and in view of the previous discussions related to the resistance expressed by Eq. (29), it can be understood that the design values expressed by Eqs. (31.a) and (b) correspond to lower values of distributions. In the expression of Eqs. (31-a) and (b), the model uncertainties that can be defined by a random variable representing the ratio between actual strengths and the predicted strengths are disregarded. Structural engineers know that these random variables are mostly larger than 1.0 and that the expressions of Eqs. (3I.a) and (b) are conservative. In view of this conservativeness, it is certain that design values for tension and bending are really lower values of the distribution of static ultimate strengths. For columns, $\left(\sigma_{u} / \sigma_{y}\right)^{*}$ is usually selected at a lower bound of experimental values when the number of experiments is few, otherwise at a fractile of 1 to 5 percents ${ }^{16), 17)}$. With this fractile, the design values defined by Eq. $(31 \cdot \mathrm{c})$ are also located at a lower value of the ultimate strength population.

The tensile and bending strengths of Eqs. $(31 \cdot a)$ and (b) are the results of elastic strength analysis, while ultimate strength analysis for elastic-perfectly plastic material gives

$$
M=\sigma_{y} Z, \quad N_{t}=\sigma_{y} A \quad \cdots \cdots \cdots \cdots \cdots \cdots(32 \cdot \mathrm{a} \sim \mathrm{b})
$$

where $Z$ is plastic section modulus. The ratio $Z / W$ is 1.08 to 1.14 for rolled sections, while the difference decreases to a few percents at most in 
the cases of such moment carrying members as plate and box girders used for bridges and cranes. It is emphasized that the tensile and bending strengths derived by elastic and plastic analyses are not much different.

It is noted that the expression of Eq. (31.c) adopted in the allowable stress design is not based on elastic analysis, but it represents static ultimate strength determined by experiments or by inelastic instability analysis. This fact is not necessarily limited to steel columns, but situation is the same for reinforced concrete columns. There are many variations of expressions of elastically analysed column strength. The simplest is

$$
N_{c}=\text { smaller of }\left(\sigma_{y} A \text { and } \pi^{2} E I / L^{2}\right)
$$

where $E I$ is bending rigidity and $L$ is length of a pinned end column. Another example is the so-called secant formula. Equation (33) predicts strengths far different from the ultimate strengths obtained through experiments. The secant formula, on the other hand, correlates reasonably well with the experimental results. Equation (33) is not specified in any design codes, whereas there are design rules that use the secant formula as the design values of column strength ${ }^{12}$.

It is noted in the allowable stress design that, whenever elastic analysis does not correlate well with experimentally obtained ultimate strength, the elastic expression is not employed but the other expressions that correlate well are being used for the strength against static failure. Equation $(31 \cdot c)$ is one of the examples. Another example is the strength of bolts and rivets at connections. This fact clearly indicates that the design for static failure in the allowable stress design codes is based on ultimate limit states. This should not be confused with the difference of structural theories to derive the expressions of resistance. Resistance may be derived based on elastic analysis or on a variety of inelastic nonlinear ultimate strength analyses. Regardless of the theoretical basis, the expressions that represent ultimate limit states are always being employed.

Resistances specified in the partial safety factor design format for steel structures are essentially the same as those specified for the allowable stress design rules and the difference, even if it exists, is very small and of no importance. The difference in concrete design is not small. This, however, is not due to the difference in format, rather it is due to the fact that the progress of structural strength analysis has not been incorporated much into the allowable stress design rules but the partial safety factor design rules have been written incorporating this progress.

\section{EVALUATION OF METHOD}

There are so many factors that have been the causes of numerous structural failures but are not directly included in $r$ and $s$ of Eq. (2). The examples are errors associated with modeling of a structure that is difficult to assess, errors due to workmanship, all sorts of human errors, and lack of knowledge such as misunderstandings of specifications. Some of these factors may be disregarded in structural design for economic reasons or by the opinions of responsible engineers whatever the reasons may be. Nevertheless, uncertainties exist that are not subject to any objective analysis at present. With the presence of those uncertainties, Eq. (7) is not sufficient to ensure target failure probability, but it is modified by introducing a factor $\nu>1$ to

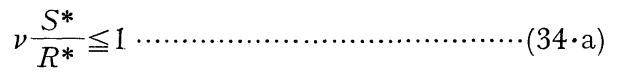

For the expression of Eq. (3·a), the design format becomes

$$
\sum_{j}\left[\left(\nu \frac{S^{*}}{R^{*}}\right)^{m}\right]_{j} \leqq 1
$$

Another reason that many engineers feel the necessity of this modification is the vague fear regarding lack of knowledge and information that were also the causes of many historical failures such as instability phenomena, brittle fracture, delayed fatigue and unpredictable size effects, all of which were not known to the responsible engineers at the time of accidents.

Since $\nu$ is introduced for uncertainty that is not subject to objective analysis, its magnitude can not be determined by a statistical consideration. Engineering judgement would be the best way to determine the value with the consideration of the performance of so many structures hitherto constructed. Most of them are surviving, while few failed. This experience may be regarded as experiments, from which information on $\nu$ may be learned.

If the uncertainty factor $v$ is combined with the expression of resistance by dividing the material strength, Eq. (34) is almost idential with most of the allowable stress design formats in conventional specifications.

In this proposed design method of Eq. (34), the uncertainty that influences the attainment of a limit state has been divided into three separate groups, which are related to resistance, load effect and subjective uncertainty. Any available statistical information to be worthy of consideration is reflected in the determination of design values related either to resistance or to load effect by Eq. (6). Hence no factor is proposed 
to consider a possibility of occurrence of overloading or lower resistance. On the other hand, the factor $\nu$ is introduced to protect the safety of structures for uncertainty that is not subject to objective analysis, at least at present, due to lack of information or knowledge and hence can not be reflected in a rational way in the design values for resistance or load effect. In this regard, the proposed design method is said to be a semi-probabilistic method with low values of resistance and high values of load effect employing a single uncertainty factor. It is noted that design values and a value of uncertainty factor generally depend on target failure probability which may be different for each limit state and load combination.

When load effects are not linear functions of their basic variables, it is obvious that

$$
\nu S\left(s_{i}\right) \neq S\left(\nu s_{i}\right)
$$

Equation (35) indicates that, generally, the uncertainty factor of the proposed design format, Eq. (34), should not directly be combined with design values of loads $^{9}$. The situation is the same for model uncertainty, which one of the basic variables of load effect may represent. The design value of this model uncertainty should not be combined with the design value of loads but it has to be determined separately and to be multiplied to the load effect evaluated with the design loads ${ }^{12)}$.

Consider a design for a static ultimate limit state in which the maximum values of external loads are well defined such as water pressure or weight of mass. Suppose that a designed structure barely satisfies Eq. (7) when load effect is evaluated under the action of these well defined maximum loads. Even under the action of these loads, the designed structure usually has reserve strength due to low design values of resistance and to the presence of the uncertainty factor. There exists, however, a possibility that, when larger loads are applied, nonlinear response due to the loss of stability may become significant and that the structure can not support the loads defined by $\nu$ times the maximum loads. This is not a matter of concern, since there is no possibility that these well defined maximum loads are exceeded. The situation is almost the same for the other loadings as long as design values are defined by Eq. $(6 \cdot \mathrm{b})$ with a small fractile that is accepted by the profession. When the value of fractile is agreed to ensure failure probability due to both overloading and lower resistance being less than a target value, overloading is no longer any worry in structural design. If overloading seems a matter of concern, the situation is simply that a still smaller fractile has to be agreed.

In the expressions of resistance of Eq. (10), one of $R_{i}$ 's, say $R_{m}$, is usually a function of only material strength designated by $r_{m}$. In most of the cases, $R_{m}$ is linear function of $r_{m}$, however there is the case that $R_{m}$ is not a linear function but nonlinear function of $r_{m}$. When $R_{m}$ is not a linear function, then

$$
\frac{R_{m}\left(v_{m}\right)}{\nu} \doteqdot R_{m}\left(\frac{v_{m}}{\nu}\right)
$$

Examples of this sort are resistances of composite girders and prestressed concrete members, in which stress resultant is not proportional to stress. Because of this, the uncertainty factor is better to be separated with the material strength and hence the allowable stress design format is preferably corrected. Material strength should not be divided by the uncertainty factor, but the resistance for a limit state has to be evaluated and then it has to be checked with the load effects and the uncertainty factor $v$ by Eq. (34). Noting that stress resultant is directly related to resistance and that there exist sections in which stress resultant is a non-linear function of stress, it is preferable in Eq. (34) to compare $S^{*}$ with $R^{*}$ in terms of stress resultants rather than stresses. This is another point that needs improvement in the existing allowable stress design codes.

In the proposed method, design values for resistance and load effect can be determined independently from each other, while numerous mathematical operations are inevitable in the second moment method.

Good correlation exists between the fractile and the failure probability and hence the fractile can be a good measure for structural reliability. The correlation is not so good in the case of safety index. Because of this difference of correlation, the proposed method is superior to the second moment method from the probabilistic standpoint. Since the design values are to be determined from available data, errors are inevitable in determination of design values and hence this superiority will be lost to certain extent. Nevertheless, this superiority of the proposed method is reasonable noting the facts that available data are the same for both methods and that the second moment method makes use of only the mean and the variance of the data, whereas the proposed method makes use of all of the information from the data including the mean and the variance if appropriate. The main feature of the proposed method is that it can make full use of statistical information in the simplest procedure.

Because of the uncertain shape of the tail 


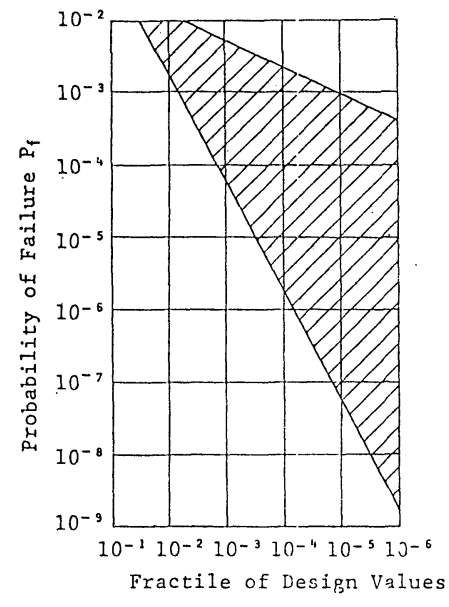

Fig. 4 Bounds of Fractile of Design Values

portion of distribution, conservative values which correspond to a smaller fractile than the target fractile may be specified as design values. Figure 4, drawn from the information of Figs. 1 and 2, shows the bounds of the fractiles corresponding to these conservative design values necessary to ensure a better accuracy of the failure probability than that attained by the second moment method within the variation of parameters of Figs. 1 and 2. For example, when the target is to ensure $P_{f}<10^{-3}$, the fractile method is superior to the second moment method if the design values can be determined within the accuracy to satisfy $\operatorname{Pr}\left(R<R^{*}\right)$ and $\operatorname{Pr}\left(S>S^{*}\right)$ between $1 / 150$ through $10^{-5}$.

The procedure to determine design values is very flexible in the proposed method. Whenever there is probabilistic reasoning or good engineering judgement to favor particular statistical distributions, this can be utilized to yield more accurate design values for their statistical definition of Eq. (6). This is especially advantageous for distributions with artificial finite bounds. Regardless of target fractile, the values at artificial bounds may be assigned for design values. The difference of available amount of statistical information can also be utilized and reflected to determine accurate design values.

The proposed method is very similar to the conventional design procedure retaining all of its simplicity and hence gradual transition to an improved design rule in the sense of failure probability seems an easy task.

\section{SUMMARY AND CONCLUDING REMARKS}

A semi-probabilistic design method is proposed by employing fractiles of distributions as a measure of structural reliability. The method defines low values of resistance and high values of load effect as design values and a single factor representing subjective uncertainty. All of statistical information are reflected in the determination of design values related either to resistance or to load effect.

Good correlation has been numerically proved to exist between the failure probability and the fractile. This indicates that the method can be a better alternative to the second moment method, retaining similar simplicity of the latter method in practical applications. The design values can be determined taking all of the advantages of available statistical information.

The format of this proposal is very similar to most of conventional allowable stress design rules which are based on elastic analyses of structures for the evaluation of load effect, and on limit states such as ultimate strength for static failure and fatigue strength for fatigue failure. The design values of dead and live loads and resistances specified in existing codes are briefly examined from a statistical point of view. It is revealed that most of the design values of conventional design rules are in good agreement with the statistical concept of this proposed method. On the other hand, some of the loads such as dead loads specified in the partial safety factor design format are subject to question. With this method, gradual transition seems an easy task to arrive at an improved design rule in the sense of consistent failure probability.

For a possible revision of the existing allowable stress design specifications, the following comments may be made from this study.

(1) The current design values of load effect and resistance should be checked and, if necessary, corrected to more consistent and appropriate values in terms of fractiles of distributions. The fractiles are not constants but depend on failure probability and hence different fractiles may be considered.

(2) If failure probability is not constant conceptually and if appropriate, different design values of load effect and resistance and the uncertainty factor should be specified for different combinations of loading and for designs for different limit states.

(3) The over-all safety factor as obvious in each design code should be separated from material strength and recognized as the uncertainty factor $\nu$. The value of this safety factor has to be re-examined, if statistical evaluation is possible for uncertainties that the factor represents. It has also to be re-examined from the view point of code calibration with the changes of design values of load effect and resistance and from 
historical experience that is increasing continuously.

(4) Structural design is to be accomplished by checking the low value of resistance, the high value of load effect and the uncertainty factor for each limit state and load combination.

(5) For ultimate limit state, the current practice of evaluation of resistance and load effect in terms of stresses should be modified to that in terms of stress resultants.

\section{ACKNOWLEDGEMENT}

The authors are grateful to Professors Manabu Ito and Hajime Okamura of the University of Tokyo for their valuable suggestions and comments given during the preparation of this paper.

\section{REFERENCES}

1) Lind, N. C.: Consistent Partial Safety Factors, Journal of the Structural Division, ASCE, Vol. 97, No. ST6, Proc. Paper 8166, pp. 16511669, June, 1971

2) Ang, A. H-S. and C. A. Cornel: Reliability Bases of Structural Safety and Design, Journal of the Structural Division, ASCE, Vol. 100, No. ST9, Proc. Paper 10777, pp. 1755-1769, Sept., 1974.

3) Ravindra M. K. and T. V. Galambos: Load and Resistance Factor Design for Steel, Journal of the Structural Division, ASCE, Vol. 104, No. ST9, Proc. Paper 14008, pp. 1337-1353, Sept., 1978.

4) Ang, A. H-S, and M. Amin: Safety Factors and Probability in Structural Design, Journal of the Structural Division, ASCE, Vol. 95, No. ST7, Proc. Paper 6667, pp. 1389-1405, July, 1969.

5) Paloheimo, E. and M. Hannus: Structural Design Based on Weighted Fractiles, Journal of the Structural Division, ASCE, Vol. 100,
No. ST7, Proc. Paper 10663, pp. 1367-1378, July, 1974.

6) Rackwitz, R.: Practical Probabilistic Approach to Design, Bulletin d'Information No. 112, CEB, pp. 13-72, July, 1976.

7) Lind, N. C.: Formulation of Probabilistic Design, Journal of the Engineering Mechanics Division, ASCE, Vol. 103, No. EM2, Proc. Paper 12861, pp. 273-284, April, 1977.

8) ACI: Building Code Requirements for Reinforced Concrete (ACI 318-77), 2nd Printing, June, 1978.

9) CEB: International System of Unified Standard Codes of Practice for Structures, Bulletin d'Information No. 124/125-E, April, 1978.

10) AASHTO: Standard Specifications for Highway Bridges, 12th Edition, AASHTO, Washington, D. C., 1977.

11) BSI: The Structural Use of Concrete, CP 110 , BSI, London, 1972.

12) BSI: Steel, Concrete and Composite Bridges, BS 5400, BSI, London, 1978.

13) Committee on Structural Safety: Structural Safety and Reliability, Japan Society of Civil Engineers, October, 1976, (In Japanese).

14) Committee on Safety and Reliability: Reliability and Safety of Steel Structures, Society of Steel Construction of Japan, Vol. 17, No. 179, pp. 1-137, February and March, 1981 (In Japanese).

15) Tokai Steel Structural Research Group: Evaluation of Strength of Steel Structural Members and Their Application to Reliability Based Design, Bridges and Foundations, November and December, 1980 (In Japanese).

16) Japan Road Association: Standard Specifications for Highway Bridges, JRA, 1980 (In Japanese).

17) Japan Society for Civil Engineers: Design Standards for Steel Railway Bridges, JSCE, 1974 (In Japanese).

(Received August 25, 1981) 